\title{
On the relation between virial coefficients and the close-packing of hard disks and hard spheres
}

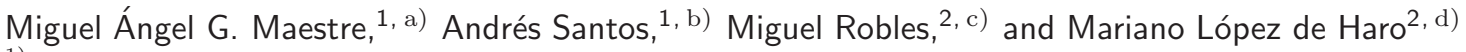 \\ 1) Departamento de Física, Universidad de Extremadura, Badajoz 06071, Spain \\ ${ }^{2)}$ Centro de Investigación en Energía, Universidad Nacional Autónoma de México (U.N.A.M.), Temixco, \\ Morelos 62580, Mexico
}

(Dated: 3 October 2018)

The question of whether the known virial coefficients are enough to determine the packing fraction $\eta_{\infty}$ at which the fluid equation of state of a hard-sphere fluid diverges is addressed. It is found that the information derived from the direct Padé approximants to the compressibility factor constructed with the virial coefficients is inconclusive. An alternative approach is proposed which makes use of the same virial coefficients and of the equation of state in a form where the packing fraction is explicitly given as a function of the pressure. The results of this approach both for hard-disk and hard-sphere fluids, which can straightforwardly accommodate higher virial coefficients when available, lends support to the conjecture that $\eta_{\infty}$ is equal to the maximum packing fraction corresponding to an ordered crystalline structure.

\section{INTRODUCTION}

The virial expansion of the equation of state of a fluid is an expansion in powers of (usually) the number density $\rho$ that reads

$$
Z(\rho, T)=1+\sum_{j=2}^{\infty} B_{j}(T) \rho^{j-1}
$$

where $B_{j}$ are the virial coefficients and $Z \equiv p / \rho k_{B} T$ is the compressibility factor, with $p, k_{B}$, and $T$ being the pressure, the Boltzmann constant, and the absolute temperature, respectively. This expansion was originally introduced by Thiesen in 1885 (Ref. 1 in connection with the equation of state of fluids at low densities. Soon afterwards, and apparently independently, it was used by Kamerlingh Onnes ${ }^{2}$ (who named the coefficients in the expansion as virial coefficients), in order to provide a mathematical representation of experimental results. The virial series was later proven to arise naturally in rigorous derivations in statistical mechanics ${ }^{3}$ whereby the $B_{j}$ turn out to be related to intermolecular interactions and are in general functions of temperature.

In the case of hard-core systems such as hard disks or hard spheres, $\stackrel{4}{=}$ the virial coefficients do not depend on temperature. In particular, the value of the second virial coefficient for hard spheres of diameter $\sigma$ in $d$ dimensions is $B_{2}=2^{d-1} v_{d} \sigma^{d}$, where $v_{d}=(\pi / 4)^{d / 2} / \Gamma(1+d / 2)$ is the volume of a $d$-dimensional hard sphere of unit diameter, a result first derived for three-dimensional hard spheres

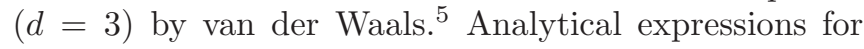

\footnotetext{
a) Electronic mail: maestre@unex.es

b) Electronic mail:

www.unex.es/eweb/fisteor/andres/

${ }^{\mathrm{c})}$ Electronic mail:

andres@unex.es

http://xml.cie.unam.mx/xml/tc/ft/mrp/

mrp@cie.unam.mx

d) Electronic mail: $\quad$ malopez@servidor.unam.mx http://xml.cie.unam.mx/xml/tc/ft/mlh/
}

TABLE I. Known virial coefficients ${ }^{35}$ for a hard-disk fluid $(d=2)$ and a hard-sphere fluid $(d=3)$.

\begin{tabular}{ccc}
\hline \hline$j$ & $b_{j}(d=2)$ & $b_{j}(d=3)$ \\
\hline 2 & 2 & 4 \\
3 & $3.12801775 \ldots$ & 10 \\
4 & $4.25785445 \ldots$ & $18.36476838 \ldots$ \\
5 & $5.3368966(2)$ & $28.22451(26)$ \\
6 & $6.36296(13)$ & $39.81515(93)$ \\
7 & $7.35186(28)$ & $53.3444(37)$ \\
8 & $8.31910(44)$ & $68.538(18)$ \\
9 & $9.27215(90)$ & $85.813(85)$ \\
10 & $10.2163(41)$ & $105.78(39)$ \\
\hline \hline
\end{tabular}

$B_{3}$ and $B_{4}$ are also available in the literature ${ }^{6-16}$ but higher virial coefficients must be computed numerically and, since this represents a non trivial task, up to now only values up to the tenth virial coefficient have been reported ${ }^{17-36}$

The virial expansion for $d$-dimensional hard-sphere systems is often expressed in terms of the packing fraction $\eta$ defined as

$$
\eta=v_{d} \rho \sigma^{d}
$$

Hence, for these systems the virial expansion of the compressibility factor is given by

$$
Z(\eta)=1+\sum_{j=2}^{\infty} b_{j} \eta^{j-1}
$$

where the (reduced) virial coefficients $b_{j} \equiv B_{j} /\left(v_{d} \sigma^{d}\right)^{j-1}$ are pure numbers. For one-dimensional hard rods, $b_{j}=1$ and so $Z(\eta)=1 /(1-\eta)$. The presently known values 35 of the virial coefficients coefficients for hard disks $(d=2)$ and hard spheres $(d=3)$ are given in Table 1 .

There are a number of controversial and open issues related to the virial expansion of hard-disk and hardsphere fluids $\frac{37}{3}$ To begin with, even in the case that many more virial coefficients for these systems were known, the 
truncated virial series for the corresponding compressibility factors would not be useful in principle for packing fractions higher than the one corresponding to the radius of convergence $\eta_{\text {conv }}$ of the whole series. Such a radius of convergence is determined by the modulus of the singularity of $Z(\eta)$ closest to the origin in the complex plane. While its actual value is not known, lower bounds are available $\frac{38.39}{}$ and existing evidence suggests that it derives from a singularity located on the real negative axis $\frac{35,40}{4 n}$ fact, one of the major reasons for trying to evaluate higher order virial coefficients is precisely the determination of the value of $\eta_{\text {conv }}$ and of the nature of the singularity giving rise to it. One should add that, although the virial series diverges for $\eta>\eta_{\text {conv }}$, given the fact that $\eta_{\text {conv }}$ seems to be located outside the positive real axis, one would expect that the compressibility factor would still be well defined for $\eta \geq \eta_{\text {conv }}$, at least in a certain range.

Coming back to the controversial and open issues, even the character of the virial expansion (either alternating or not) is still unknown. So far, all the available virial coefficients for these systems are positive, but results from higher dimensions suggest that this feature might not be true for higher virial coefficients $\underline{\underline{35}}$ Finally, the evidence coming from approximate equations of state obtained through the knowledge of the limited number of virial coefficients via various series acceleration methods, such as Padé or Levin approximants, indicates that the freezing transition observed in computer simulations does not show up as a singularity in these equations of state $\underline{\underline{41}}$ As a matter of fact, while it is quite plausible that $Z(\eta)$ presents a singularity at the freezing density $\eta_{f}, \underline{42-44}$ the virial coefficients (or even their asymptotic behavior) do not seem to yield any information concerning the freezing transition at $\eta_{f} \underline{\underline{42}}$ This might be related to the fact that $\lim _{\eta \rightarrow \eta_{f}^{-}} Z(\eta)=$ finite.

Since the compressibility factor of hard-disk and hardsphere fluids both for the stable and metastable fluid phases is a monotonically increasing function of the packing fraction,, 37 one may reasonably wonder at which packing fraction $\eta=\eta_{\infty}$ the analytical continuation of the compressibility factor diverges, namely one wants to find the value of $\eta_{\infty}$ such that

$$
\lim _{\eta \rightarrow \eta_{\infty}} Z(\eta)=\infty .
$$

Clearly, $\eta_{\infty}$ may not be bigger than the maximum packing fraction $\eta_{\max }$ that is geometrically possible (the socalled Kepler's problem). Therefore, one must have

$$
\eta_{\text {conv }} \leq \eta_{\infty} \leq \eta_{\max } .
$$

The value of $\eta_{\max }$, at least for not too high dimensionalities $d$, corresponds to an ordered crystalline structure. In Table II we provide the values of $\eta_{\max }$ for $d=2-8$.

More than a decade ago, by studying the singularities of Padé approximants constructed from the virial series for hard disks and hard spheres, Sanchez ${ }^{46}$ came to the
TABLE II. Values of $\eta_{\max }$ for $d=2-8, \frac{45}{=}$

\begin{tabular}{lcc}
\hline \hline$d$ & Exact & Numerical \\
\hline 2 & $\frac{1}{6} \pi \sqrt{3}$ & 0.9069 \\
3 & $\frac{1}{6} \pi \sqrt{2}$ & 0.7405 \\
4 & $\frac{1}{16} \pi^{2}$ & 0.6168 \\
5 & $\frac{1}{30} \pi^{2} \sqrt{2}$ & 0.4652 \\
6 & $\frac{1}{144} \pi^{3} \sqrt{3}$ & 0.3729 \\
7 & $\frac{1}{105} \pi^{3}$ & 0.2953 \\
8 & $\frac{1}{384} \pi^{4}$ & 0.2537 \\
\hline \hline
\end{tabular}

conclusion that such singularities were related to crystalline close-packing in these systems. Other authors $\underline{47}-61$ have also conjectured that

$$
\eta_{\infty}=\eta_{\max }
$$

It is interesting to note that this conjecture was already suggested by Korteweg 62 and Boltzmann ${ }^{63}$ in the late 1800 s.

On the other hand, the conjecture (1.6) has not been free from criticism $\frac{42}{2}$ and some authors $\underline{\underline{64}} \underline{68}$ have conjectured that $\eta_{\infty}=\eta_{\mathrm{rcp}}$, where $\eta_{\mathrm{rcp}}$ (approximately equal to 0.82 and 0.64 for hard disks and hard spheres, respectively ${ }^{69}$ ) is the random close-packing fraction. For a thorough account of proposed equations of state, including those enforcing $\eta_{\infty}=\eta_{\max }$ or $\eta_{\infty}=\eta_{\mathrm{rcp}}$, the reader is referred to Ref. 70 .

In his original study, Sanchez used a Padé analysis with the then most recent available values of the first eight virial coefficients given by Janse van Rensburg $\underline{\underline{29}}$ More recently, Sanchez and Lee ${ }^{71}$ found that, using the ninth and tenth virial coefficients 35 for a hard-sphere fluid, the corresponding Padé approximants remained finite at $\eta=\eta_{\max }$. However, taking into account such coefficients, they constructed a new approximate equation of state that diverges at that packing fraction. In this regard, the question arises as to whether using the available information on virial coefficients one can reach a more definite conclusion. More generally, one could ask whether there may be a systematic method to improve the estimation of $\eta_{\infty}$ as more virial coefficients become available.

The paper is organized as follows. In Sec. II we perform an analysis of the singularities of the compressibility factors obtained from the different Padé approximants constructed from the known virial coefficients of the harddisk and hard-sphere fluids. This is followed in Sec. III by the introduction of inverse representations of the virial series and their connection with the computation of $\eta_{\infty}$, the results being presented in Sec.IV] The paper is closed in Sec. $\mathbf{V}$ with some concluding remarks. 


\section{PADÉ APPROXIMANTS AND SINGULARITIES IN THE COMPRESSIBILITY FACTOR}

In general, given the series

$$
S(z)=\sum_{j=0}^{\infty} a_{j} z^{j},
$$

the notion behind a Padé approximant is the replacement of the series by a ratio of two polynomials, namely

$$
S(z) \approx P_{N}^{M}(z)=\frac{\sum_{j=0}^{M} \alpha_{j} z^{j}}{\sum_{j=0}^{N} \beta_{j} z^{j}},
$$

where, without loss of generality, one may take $\beta_{0}=1$. The remaining $N+M+1$ coefficients are chosen in such a way that the Taylor series expansion of $P_{N}^{M}(z)$ exactly yields the first $N+M+1$ terms of the power series $S(z)$. On the other hand, the difference $N-M$ of the degrees of the two polynomials may be taken at will. A more detailed treatment of Padé approximants may be found in Ref. 72 .

In the usual application of Padé approximants for the equation of state of hard-sphere fluids, the compressibility factor $Z$ is approximated by

$$
Z(\eta) \approx P_{N}^{M}(\eta)=\frac{1+\sum_{j=1}^{M} \alpha_{j} \eta^{j}}{1+\sum_{j=1}^{N} \beta_{j} \eta^{j}},
$$

where, since $\lim _{\eta \rightarrow 0} Z(\eta)=1$, one sets $\alpha_{0}=1$. The other $N+M$ coefficients $\left\{\alpha_{j}, j=1, \ldots, M\right\}$ and $\left\{\beta_{j}, j=\right.$ $1, \ldots, N\}$ are determined with the values of the virial coefficients $\left\{b_{j}, j=2, \ldots N+M+1\right\}$. For a given value of $g \equiv N+M$, there are in principle $g$ independent approximants (corresponding to $N=1, \ldots, g$ ), because the case $(N, M)=(0, g)$ is not a Padé approximant but rather a truncated series. We will use the term 'Padé degree' for $g=N+M$. Given the known values of $b_{j}$ (see Table 【), the highest value of the Padé degree is $g=9$. The Padé approximants (2.3) are not only used to get the equation of state but also to predict values of unknown virial coefficients $\underline{\underline{73}, 74}$

Both the radius of convergence $\eta_{\text {conv }}$ of the virial series and the value of $\eta_{\infty}$ when $Z$ is approximated by (2.3) are determined by the nature of the singularities of the approximant. The modulus of the complex root of $1+$ $\sum_{j=1}^{N} \beta_{j} \eta^{j}$ closest to the origin gives $\eta_{\text {conv }}$, while $\eta_{\infty}$ is identified with the smallest real positive root.

Due to our interest in obtaining the value of $\eta_{\infty}$, we have carried out a systematic analysis of the location of the real and positive pole of $P_{N}^{M}(\eta)$ closest to the origin. For each Padé degree $g \leq 9$ we have considered all the associated $g$ Padé approximants, namely a total of $\sum_{g=1}^{9} g=45$ approximants, and located their poles. In some instances, the corresponding approximant either has no real and positive poles or these are located beyond $\eta=1$. In the rest of the cases, if more than one

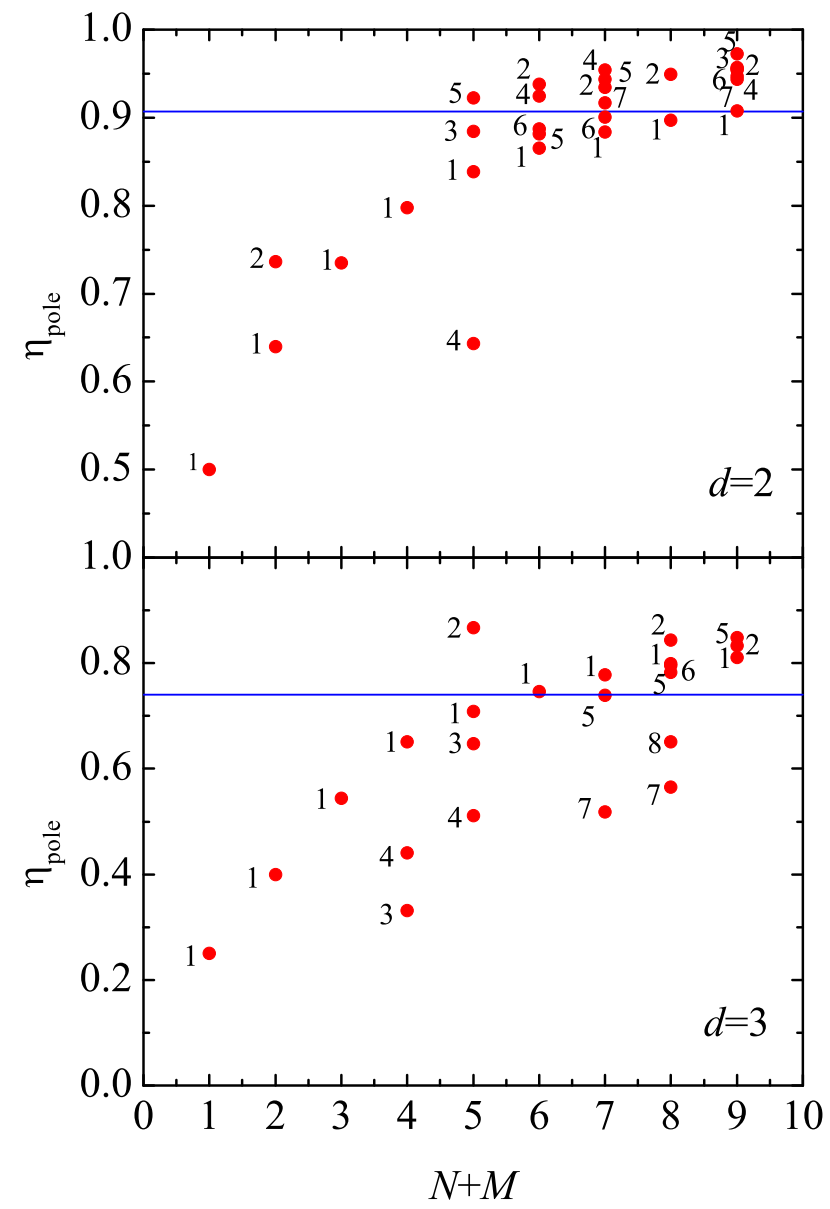

FIG. 1. Value of the real and positive pole closest to the origin for the different Padé approximants of the form (2.3) as a function of the Padé degree $g=N+M$. The number placed next to each circle indicates the degree $N$ of the polynomial in the denominator of the corresponding Padé approximant. The horizontal lines correspond to $\eta=\eta_{\max }$.

real and positive pole existed in the range $0<\eta<1$, we have focused on the smallest one of them. Moreover, we have discarded a pole when it practically coincided with a zero of the approximant. The results of this analysis are shown in Fig. 1 .

As can be seen in this figure, only $29(d=2)$ or 23 $(d=3)$ out of the 45 approximants possess a real and positive pole in the range $0<\eta<1$. The Padé approximants with $N=1$ have a real pole at $\eta_{\text {pole }}=b_{q} / b_{g+1}$ and this is the only pole for a few values of $g$. Also worth pointing out is the fact that the real and positive singularity closest to the origin exhibits an important dispersion for a given Padé degree $g$, something particularly noticeable for $g=8$ and $d=3$. Moreover, for $g \geq 5$ the value of the poles may even be higher than $\eta_{\max }$, which is clearly unphysical.

In order to check on the robustness of the method of analysis, we have carried out the same analysis for the cases of $g=8$ and $g=9$, this time varying the values of the two last known virial coefficients by $5 \%$. More specif- 


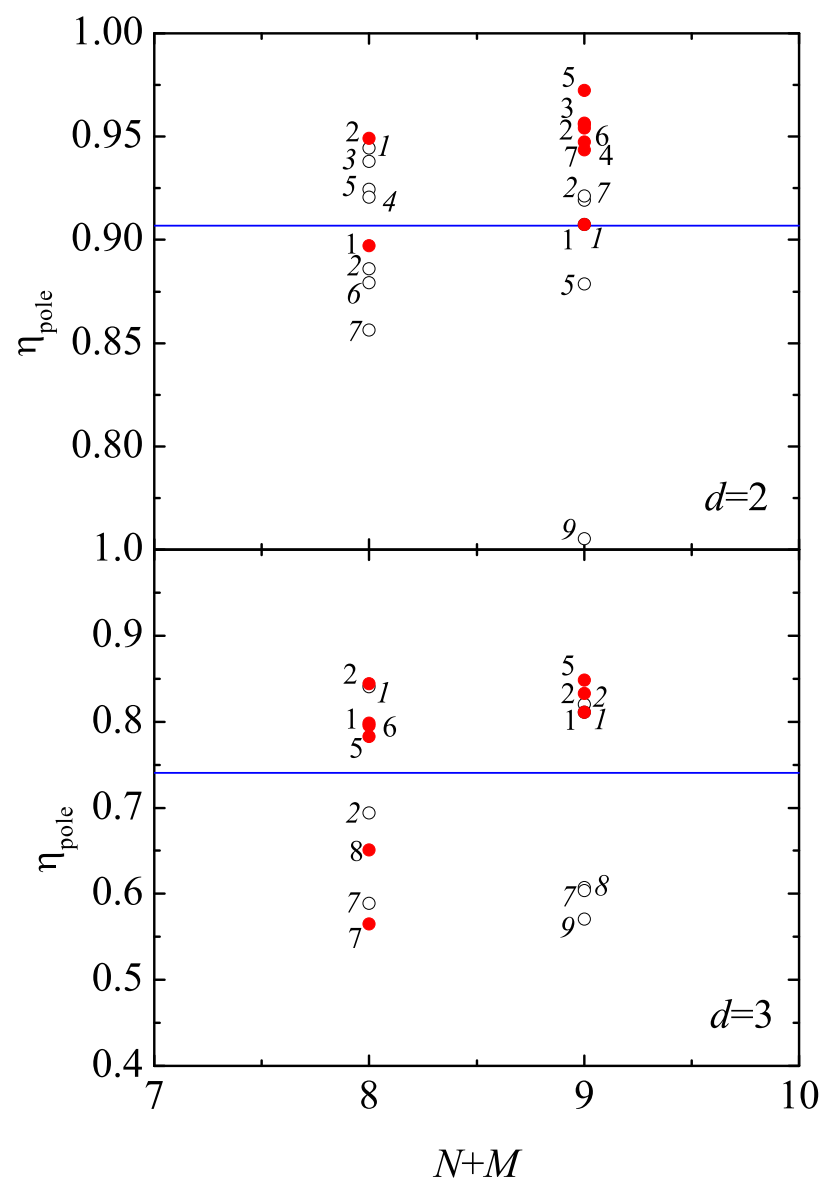

FIG. 2. Value of the real and positive pole closest to the origin for $g=8$ and $g=9$. Filled circles correspond to the values obtained from the use of the coefficients $b_{9}$ and $b_{10}$ given in Table [1. while the open circles have been obtained using the values $\left(b_{9}, b_{10}\right)=(8.80,9.71)$ for $d=2$ and $\left(b_{9}, b_{10}\right)=(81.52,100.5)$ for $d=3$. The number placed next to each circle (in italics for open circles) indicates the degree $N$ of the polynomial in the denominator of the corresponding Padé approximant. The horizontal lines correspond to $\eta_{\max }$.

ically, instead of the values of $b_{9}$ and $b_{10}$ given in Table 1. we have taken $\left(b_{9}, b_{10}\right)=(8.80,9.71)$ for $d=2$ and $\left(b_{9}, b_{10}\right)=(81.52,100.5)$ for $d=3$. The consequence of such replacement is an important variation in the character and location of the poles, as shown in Fig. 2 including the appearance of new poles and the disappearance of earlier ones. An exception is the pole $\eta_{\text {pole }}=b_{9} / b_{10}$ corresponding to $N=1$ and $g=9$, which obviously is not affected by a common factor multiplying both $b_{9}$ and $b_{10}$. Given this generally great sensitivity of the poles on the values of the virial coefficients, it does not seem wise to rely on the previous analysis in order to get good estimates of $\eta_{\infty}$. Instead, in Sec. III we will follow a different route.

\section{INVERSE REPRESENTATIONS OF THE VIRIAL SERIES}

We have seen that the use of the direct Padé approximants of the compressibility factor $Z$ is not reliable for the purpose of determining $\eta_{\infty}$. This is perhaps not surprising since, as $\mathrm{McCoy}^{37}$ has pointed out in his recent book (and we quote), "there is no reason to expect that simple model equations of state which use the density as the independent variable will capture the true physics of hard particle systems (or indeed of any real system)." Therefore, an alternative approach is called for. We begin by introducing an equivalent form of the virial series (1.3), namely

$$
\widetilde{p}(\eta) \equiv v_{d} \sigma^{d} \frac{p}{k_{B} T}=\eta+\sum_{j=2}^{\infty} b_{j} \eta^{j} .
$$

Note that $\widetilde{p}=\eta Z$. Following an idea of Sanchez,, 46 we may formally invert the series in (1.3), leading to

$$
\eta(Z)=\sum_{j=1}^{\infty} e_{j}(Z-1)^{j} .
$$

Here we propose a similar inversion of the series (3.1) in the form

$$
\eta(\widetilde{p})=\widetilde{p}+\sum_{j=2}^{\infty} c_{j} \widetilde{p}^{j} .
$$

The coefficients $\left\{c_{j}\right\}$ and $\left\{e_{j}\right\}$ are (nonlinear) combinations of the virial coefficients $\left\{b_{j}\right\}$. In fact, the determination of $c_{j}$ and $e_{j}$ requires that one previously knows $j$ and $j+1$ virial coefficients, respectively. Notice that, while going from Eq. (1.3) to Eq. (3.1) or vice versa is a trivial task, the same is not true when considering the inverse developments (3.2) and (3.3). Further, the condition (1.4) to obtain $\eta_{\infty}$ is equivalent to

$$
\eta_{\infty}=\lim _{Z \rightarrow \infty} \eta(Z)=\lim _{\tilde{p} \rightarrow \infty} \eta(\widetilde{p}) .
$$

The form 3.2 was the one that Sanchez used 46 to estimate the value of $\eta_{\infty}$. However, the true thermodynamic variable, along with the density (hereby represented by $\eta$ ), is the pressure (hereby represented by $\widetilde{p}$ ), and not the ratio pressure over density (given by $Z$ ). Therefore, in our view the form (3.3) has a clearer physical meaning than (3.2). In this respect, it is interesting to note that Hamad 68.75 proposed for hard-disk and hard-sphere fluids an approximate equation of state of the form

$$
\eta(\widetilde{p})=\frac{\widetilde{p}}{1+b_{2} \widetilde{p}-k_{1} \widetilde{p} \ln \frac{1+k_{2} \widetilde{p}}{1+k_{3} \widetilde{p}}},
$$

with $k_{1}=\left(b_{2}^{2}-b_{3}\right) /\left(k_{2}-k_{3}\right)$ and where $k_{2}$ and $k_{3}$ were obtained by a fitting method. According to Eq. (3.5), $\eta_{\infty}=\left[b_{2}-k_{1} \ln \left(k_{2} / k_{3}\right)\right]^{-1}$, which turns out to be close to the respective random close-packing values $\underline{68}$ 


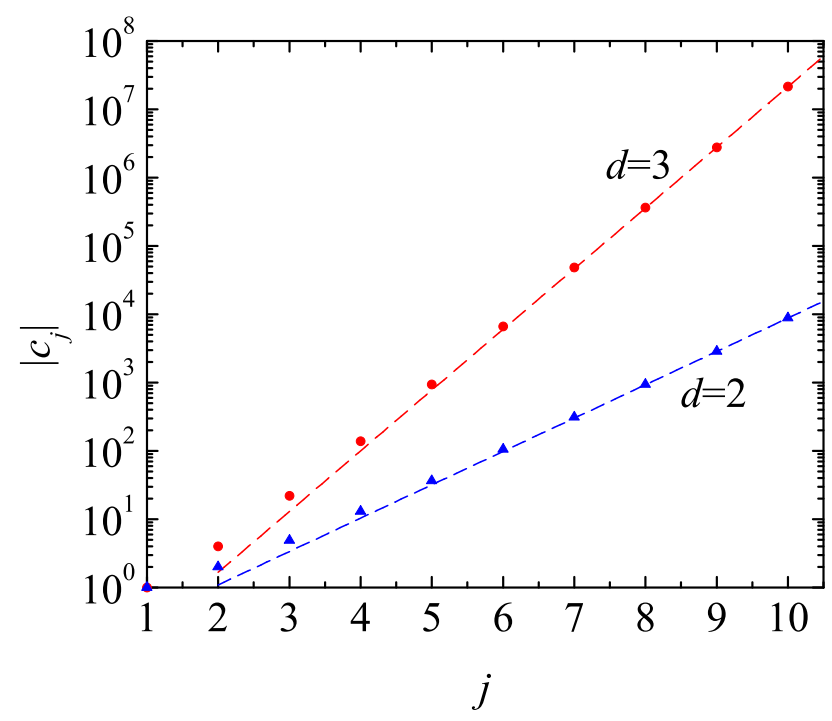

FIG. 3. Semi-logarithmic plot of the absolute value of the inverse virial coefficients $c_{j}$ for $j \leq 10$. The dashed lines represent the exponential fit $\left|c_{j}\right| \approx \overline{c_{9}}\left(\left|c_{10}\right| / c_{9}\right)^{j-9}$.

There is an additional argument in favor of expressing the equation of state in the form $\eta=\eta(\widetilde{p})$. For onedimensional systems of particles interacting through an arbitrary potential $\varphi(r)$ (restricted to nearest neighbors) there is an exact statistical-mechanical solution. $\underline{76}-\underline{78}$ In this solution, the equation of state is given by ${ }^{79}$

$$
\rho(p, T)=\frac{\Omega_{0}(p / k T, T)}{\Omega_{1}(p / k T, T)}
$$

where

$$
\Omega_{s}(x, T) \equiv \int_{0}^{\infty} d r r^{s} e^{-x r} e^{-\varphi(r) / k T} .
$$

Thus, at a given temperature, the density is an explicit function of the pressure. In general (the hard-rod case is an exception), it is not possible to invert Eq. (3.6) to express the pressure as an explicit function of density or the density as an explicit function of $Z$. The functional form (3.6) may be easily extended to the case of mixtures ${ }^{80}$

We have obtained the values of the inverse virial coefficients $c_{j}$ for $2 \leq j \leq 10$ and $e_{j}$ for $1 \leq j \leq 9$ from the knowledge of the first ten virial coefficients listed in Table【. Here we give some details about the coefficients $c_{j}$. It is observed that they alternate sign $\left(c_{2}=-b_{2}\right.$ being negative) and their absolute values grow almost exponentially with $j$. The results are graphically displayed in Fig. 3

For further use, it is interesting to provide the expressions of the coefficients $c_{9}$ and $c_{10}$ when $b_{2}-b_{8}$ are given by Table $\square$ but $b_{9}$ and $b_{10}$ remain free. For hard disks, one gets

$$
\begin{gathered}
c_{9}=2.86 \times 10^{3}\left(1-3.49 \times 10^{-4} b_{9}\right), \\
c_{10}=-8.97 \times 10^{3}\left(1-2.45 \times 10^{-3} b_{9}+1.11 \times 10^{-4} b_{10}\right) .
\end{gathered}
$$

Since $b_{9}$ and $b_{10}$ are of the order of 10 , it is clear that neither $c_{9}$ nor $c_{10}$ are strongly affected by the precise values of $b_{9}$ and $b_{10}$. This effect is even much more pronounced in the case of hard spheres, where

$$
\begin{gathered}
c_{9}=2.78 \times 10^{6}\left(1-3.60 \times 10^{-7} b_{9}\right), \\
c_{10}=-2.15 \times 10^{7}\left(1-2.05 \times 10^{-6} b_{9}+4.66 \times 10^{-8} b_{10}\right) .
\end{gathered}
$$

Taking into account that both $b_{9}$ and $b_{10}$ are of order $10^{2}$, one sees that the actual values of $c_{9}$ and $c_{10}$ are very weakly influenced by $b_{9}$ and $b_{10}$. The same process can be repeated for $c_{11}$ and $c_{12}$, this time using the values of $b_{9}$ and $b_{10}$ listed in Table \ but leaving $b_{11}$ and $b_{12}$ free. The results for hard disks are

$$
c_{11}=2.72 \times 10^{4}\left(1-3.67 \times 10^{-5} b_{11}\right),
$$

$c_{12}=-8.53 \times 10^{4}\left(1-3.05 \times 10^{-4} b_{11}+1.17 \times 10^{-5} b_{12}\right)$.

Analogously, for hard spheres one finds

$$
\begin{gathered}
c_{11}=1.68 \times 10^{8}\left(1-5.96 \times 10^{-9} b_{11}\right), \\
c_{12}=-1.32 \times 10^{9}\left(1-3.92 \times 10^{-8} b_{11}+7.55 \times 10^{-10} b_{12}\right) .
\end{gathered}
$$

Therefore, as $j$ increases, the values of $c_{j}$ become less and less sensitive to the actual values of the virial coefficients $b_{j-1}$ and $b_{j}$, this effect being much more important for hard spheres than for hard disks. This implies that one could get good estimates of $c_{11}$ and $c_{12}$ (especially for hard spheres) even with poor estimates of the unknown virial coefficients $b_{11}$ and $b_{12}$. We will come back to this point later.

Once we have introduced the representations (3.2) and (3.3), the next step is to compute their corresponding Padé approximants. These read

$$
\eta(Z) \approx(Z-1) P_{N}^{N-1}(Z-1)
$$

and

$$
\eta(\widetilde{p}) \approx \widetilde{p} P_{N}^{N-1}(\widetilde{p}) .
$$

Now one can determine $\eta_{\infty}$ using Eq. (3.4). Since $\eta_{\infty}$ must be finite and different from zero, we must restrict ourselves to diagonal Padé approximants, in contrast with what occurs with the direct approximants (2.3). Therefore,

$$
\eta_{\infty}(N)=\frac{\alpha_{N-1}}{\beta_{N}},
$$

where, following the notation of Eq. (2.2), $\alpha_{N-1}$ and $\beta_{N}$ are the coefficients of the terms of the highest degree in the numerator and denominator of the approximant, respectively. In Eq. (3.18) the notation $\eta_{\infty}(N)$ indicates that, in principle, the value so obtained may depend on 
$N$. One would expect that the true value of $\eta_{\infty}$ would be $\eta_{\infty}=\lim _{N \rightarrow \infty} \eta_{\infty}(N)$.

Since $b_{1}=1$, in the case of Eq. (3.16) we have to determine $2 N$ coefficients using $e_{1}, \ldots e_{2 N}$ (or, equivalently, $\left.b_{2}, \ldots b_{2 N+1}\right)$. On the other hand, in order to use Eq. (3.17), $2 N-1$ coefficients must be determined from $c_{2}, \ldots c_{2 N}$ (or, alternatively, from $b_{2}, \ldots b_{2 N}$ ). We recall that only ten virial coefficients are presently known, so the highest $N$ that in principle may be taken in connection with Eqs. (3.16) and (3.17) is 4 and 5, respectively. This restriction may be removed at the expense of including estimates of the first few virial coefficients beyond the tenth.

\section{RESULTS}

The results for $\eta_{\infty}(N)$, derived from the different Padé approximants as given by Eq. (3.16) (with $N=1,2,3,4$ ) and the use of Eq. (3.18) are shown in Fig. 4. Those pertaining to Eq. (3.17) (this time with $N=1,2,3,4,5$ ) appear in Fig. 5

As clearly seen from these two figures, the behavior of the inverse representations is much more regular than the one displayed in Fig. 1. This strongly supports the notion that they are more adequate if one wants to get a reliable value for $\eta_{\infty}$. Note that the results derived from Eq. (3.17), (cf. Fig. 5) show a smooth behavior and that the estimate $\eta_{\infty}(N)$ increases slowly with $N$. In the three-dimensional case, it is not clear whether $\eta_{\infty}(N)$ converges to the close-packing value $\eta_{\max }$ for $N \rightarrow \infty$ or to the random close-packing value $\eta_{\mathrm{rcp}} \simeq 0.64$. However, the very good fit obtained with a law of the form $\eta_{\infty}(N)=\eta_{\max }-a e^{-b N}$ suggests that the values obtained up to $N=5$ are not incompatible with the result $\lim _{N \rightarrow \infty} \eta_{\infty}(N)=\eta_{\max }$. In the two-dimensional case the approach of $\eta_{\infty}(N)$ to $\eta_{\max }$ is much clearer than in the three-dimensional case, the value of $\eta_{\infty}(5)$ being even slightly larger than $\eta_{\text {rcp }} \simeq 0.82$. This gives further support to the correctness of the conjecture (1.6).

Now we turn to Fig. 4. Although the representation (3.16) leads to one point less in the graph than Eq. (3.17) and does not make use of $b_{10}, \eta_{\infty}(4)$ is remarkably close to $\eta_{\max }$, especially in the case $d=2$. This could be just a coincidence and it is conceivable that, once $b_{11}$ became available and the point corresponding to $N=5$ were included, $\eta_{\infty}(5)$ would turn out to be greater than $\eta_{\max }$. In fact, the fit to a law $\eta_{\infty}(N)=\eta_{\max }-a e^{-b N}$ is much poorer in this instance. Further, we have found that the results for $\eta_{\infty}(N)$ obtained from Eq. (3.16) are more sensitive to variations of the values of the virial coefficients than those obtained from Eq. (3.17).

In view of the above, and besides the physical reasons we alluded to before, we find that the pressure representation seems to be the most reliable one for estimating the true value of $\eta_{\infty}$. On the other hand, it seems also clear that, strictly speaking, the knowledge of the first ten virial coefficients is not enough to decide whether

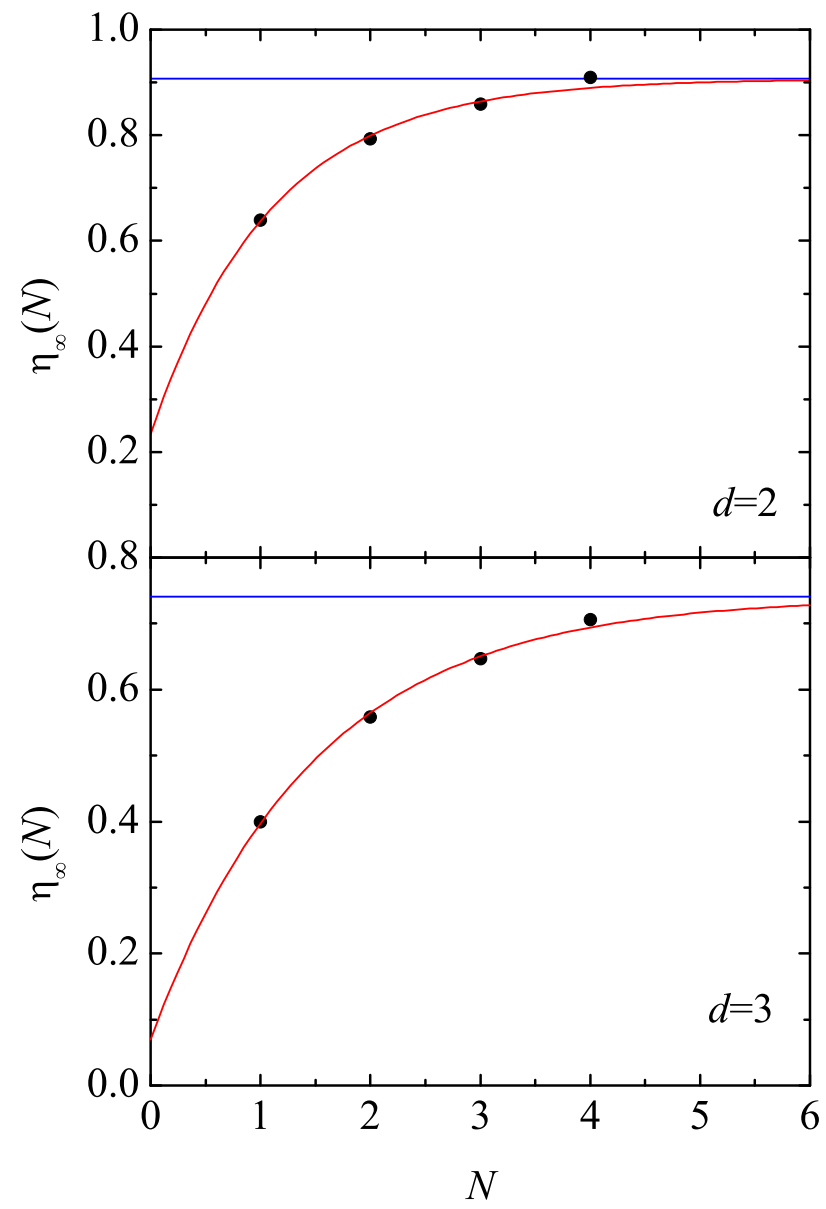

FIG. 4. Estimates of $\eta_{\infty}$ derived from Eq. 3.16). The horizontal lines correspond to $\eta_{\max }$. The curves correspond to the fit $\eta_{\infty}(N)=\eta_{\max }-0.67 e^{-0.92 N}(d=2)$ and $\eta_{\infty}(N)=$ $\eta_{\max }-0.67 e^{-0.67 N}(d=3)$.

$\eta_{\infty}=\eta_{\max }$ or $\eta_{\infty}=\eta_{\mathrm{rcp}}$ in the three-dimensional case.

In order to look into the performance of the representation (3.17) in more detail, we have carried out a test of robustness similar to the one made in connection with the poles of the conventional approximants in Fig. 2 In this case, however, rather than considering for each $N$ only an error of $\pm 5 \%$ in the values of the highest virial coefficients, we take an error of $\pm 100 \%$ in $b_{2 N}$. The results of this procedure are also shown in Fig. [5, where it is clear that as one increases $N$ the effect of the error becomes less pronounced, to the point that for $N=5$ and $d=3$ it becomes practically unnoticeable. This means that one cannot rely on the representation (3.17) for getting estimates of unknown virial coefficients. On the other hand, its introduction was not made for that purpose but rather for obtaining good estimates of $\eta_{\infty}$.

In this regard, albeit on a more speculative basis, for the sake of going beyond the limit imposed by the value $N=5$ one can incorporate into the procedure estimates of unknown higher virial coefficients $b_{11}, b_{12}, \ldots$ Given the robustness of the results as discussed above, one 


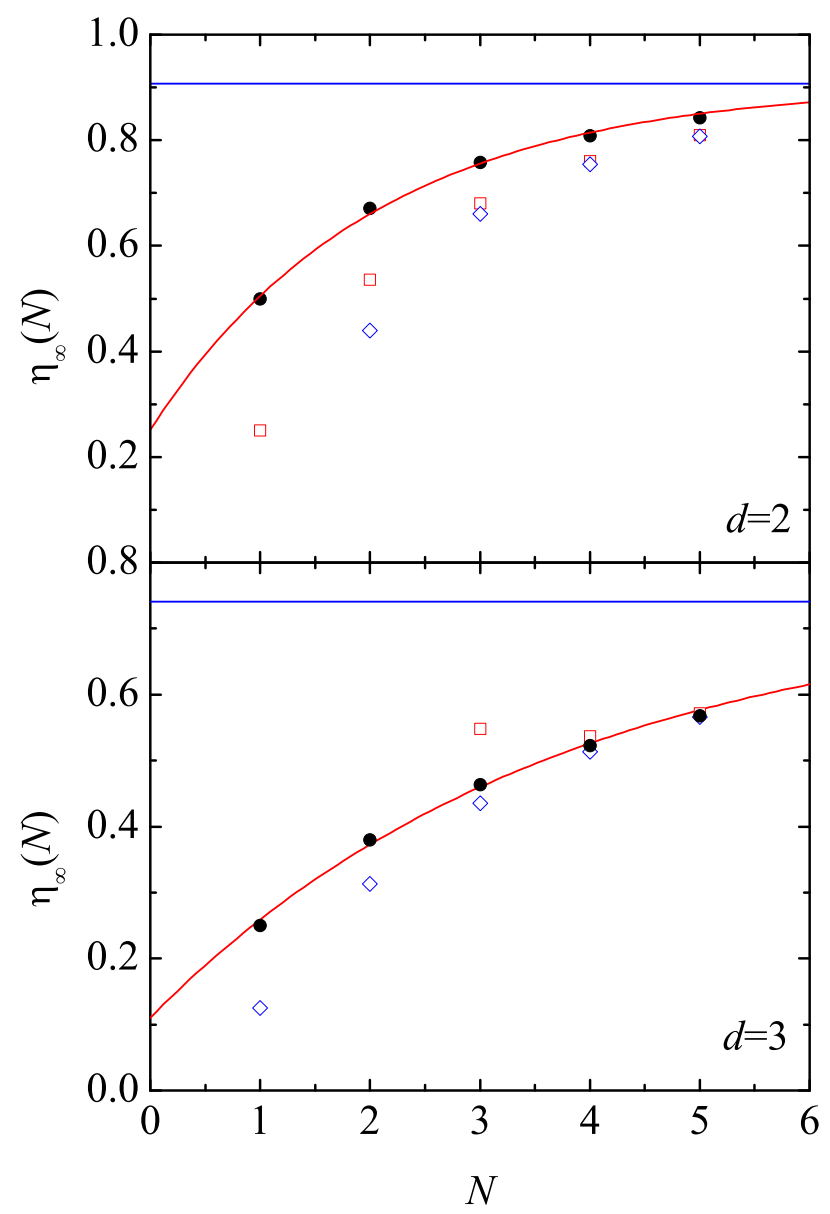

FIG. 5. Estimates of $\eta_{\infty}$ derived from Eq. (3.17). The horizontal lines correspond to $\eta_{\max }$. The curves correspond to the fit $\eta_{\infty}(N)=\eta_{\max }-0.65 e^{-0.49 N}(d=2)$ and $\eta_{\infty}(N)=$ $\eta_{\max }-0.63 e^{-0.27 N}(d=3)$. The circles, squares, and diamonds correspond to taking for $b_{2 N}$ its correct value given in Table \ zero, and twice the correct value, respectively.

would expect that the effect of errors in such estimates on $\eta_{\infty}(N)$ for $N$ somewhat greater than 5 would be weak. The idea that this is indeed the case goes back to Eqs. (3.8) - (3.15). Let us specialize here to $d=3$. From Eqs. (3.10) and (3.11) one obtains

$$
\begin{aligned}
\alpha_{4}= & \frac{120.505}{1-1.30 \times 10^{-3} b_{9}}\left(1-2.64 \times 10^{-3} b_{9}\right. \\
& \left.-1.068 \times 10^{-6} b_{9}^{2}+4.40 \times 10^{-4} b_{10}\right), \\
\beta_{5}= & \frac{211.567}{1-1.30 \times 10^{-3} b_{9}}\left(1-2.65 \times 10^{-3} b_{9}\right. \\
& \left.-1.31 \times 10^{-6} b_{9}^{2}+4.79 \times 10^{-4} b_{10}\right)
\end{aligned}
$$

for the coefficients of highest degree in the numerator and denominator of the Padé approximant (3.17) with $N=5$. Since $b_{9} \sim b_{10} \sim 10^{2}$, it turns out that $\alpha_{4}$ and $\beta_{5}$ are hardly affected by the precise values of $b_{9}$ and $b_{10}$. But, because of a partial cancelation of terms, this
TABLE III. Estimated virial coefficients ${ }^{35} b_{11}-b_{16}$ for a harddisk fluid $(d=2)$ and a hard-sphere fluid $(d=3)$.

\begin{tabular}{ccc}
\hline \hline$j$ & $b_{j}(d=2)$ & $b_{j}(d=3)$ \\
\hline 11 & 11.15 & 128 \\
12 & 12.08 & 153 \\
13 & 13.03 & 182 \\
14 & 13.93 & 215 \\
15 & 14.91 & 247 \\
16 & 15.86 & 279 \\
\hline \hline
\end{tabular}

influence is still weaker in the case of the ratio $\alpha_{4} / \beta_{5}$, namely

$$
\begin{aligned}
\eta_{\infty}(5) \simeq & 0.5696\left(1+4.6 \times 10^{-6} b_{9}+2.5 \times 10^{-7} b_{9}^{2}\right. \\
& \left.-3.9 \times 10^{-5} b_{10}\right) .
\end{aligned}
$$

When replacing the known values $b_{9}=85.813$ and $b_{10}=$ 105.78 one gets $\eta_{\infty}(5)=0.5696 \times(1-0.002)$, which only differs $0.2 \%$ from the value obtained by setting $b_{9}=b_{10}=$ 0 .

Let us repeat the same process in the case $N=6$. It can be checked that Eqs. (3.14) and (3.15) yield

$$
\begin{aligned}
\alpha_{5}= & \frac{291.651}{1-2.95 \times 10^{-4} b_{11}}\left(1-5.98 \times 10^{-4} b_{11}\right. \\
& \left.-6.03 \times 10^{-8} b_{11}^{2}+1.11 \times 10^{-4} b_{12}\right) \\
\beta_{6}= & \frac{482.486}{1-2.95 \times 10^{-4} b_{11}}\left(1-5.99 \times 10^{-4} b_{11}\right. \\
& \left.-6.98 \times 10^{-8} b_{11}^{2}+1.18 \times 10^{-4} b_{12}\right), \\
\eta_{\infty}(6) \simeq & 0.6045\left(1+5.1 \times 10^{-7} b_{11}+9.4 \times 10^{-9} b_{11}^{2}\right. \\
& \left.-7.1 \times 10^{-6} b_{12}\right) .
\end{aligned}
$$

Inserting the estimated values $b_{11} \simeq 128, b_{12} \simeq 153$ (see Table【II) we obtain $\eta_{\infty}(6)=0.6045 \times(1-0.0008)$, which deviates less than $0.1 \%$ from the value corresponding to $b_{11}=b_{12}=0$. Considering the above as an illustrative example, it is not unreasonable to expect that, as $N$ increases, the value of $\eta_{\infty}(N)$ becomes less and less sensitive to the actual values of the virial coefficients $b_{j}$ with $j \lesssim 2 N$.

With the previous assertion in mind, here we will go up to $N=8$ taking the values of $b_{11}-b_{16}$ as estimated by Clisby and McCoy 35 from Padé approximants. These estimates are given in Table III. The corresponding results for $\eta_{\infty}(N)$ are shown in Fig. 6. As was done in Fig. 5. in order to illustrate representative outcomes, we have also included in Fig. 6 for $N=6,7$, and 8 the points obtained when the values of $b_{11}$ and $b_{12}$ from Table III for $d=2(d=3)$ have an error of $\pm 10 \%( \pm 20 \%)$, those of $b_{13}$ and $b_{14}$ an error of $\pm 20 \%( \pm 40 \%)$, and those of $b_{15}$ and $b_{16}$ an error of $\pm 30 \%( \pm 60 \%)$. Once more, irrespective of the errors, one can not distinguish the three points obtained in this way with the same $N \geq 6$ in the case $d=3$. Apart from this, perhaps the most interesting 


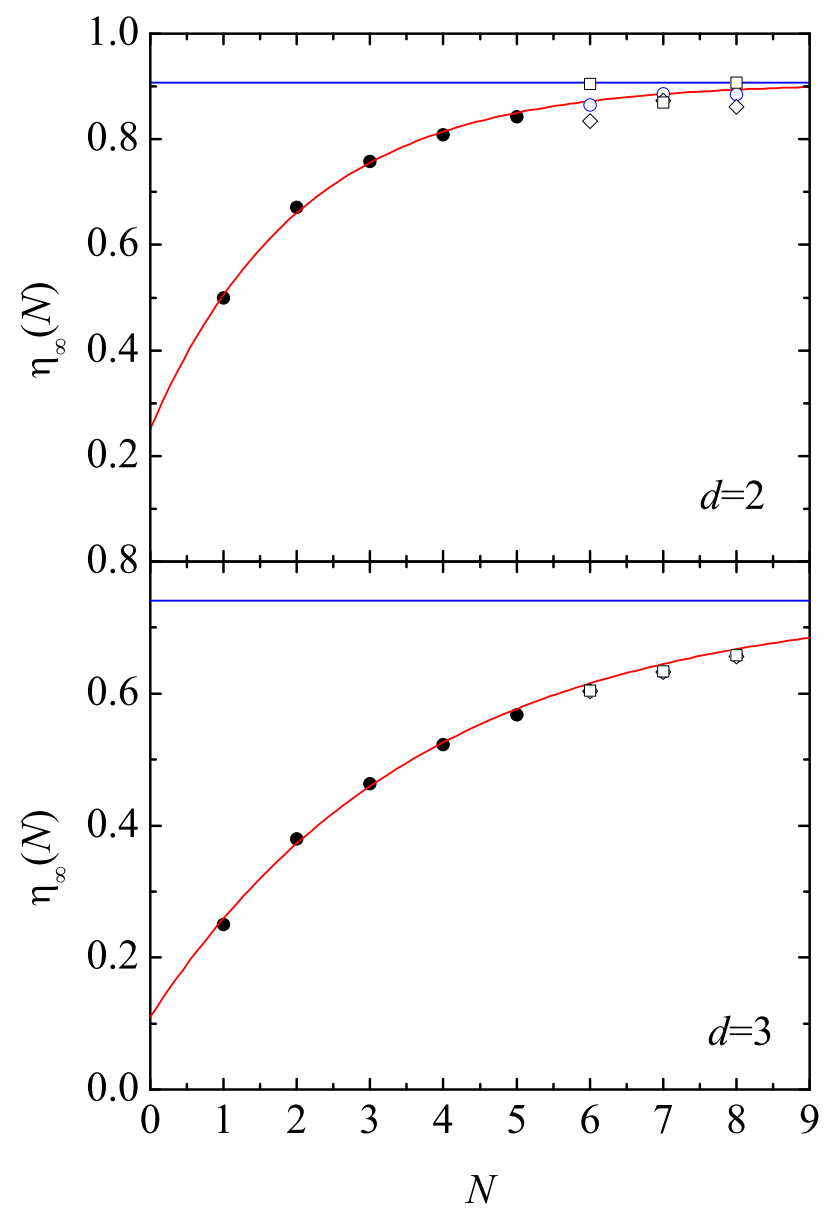

FIG. 6. Estimates of $\eta_{\infty}$ derived from Eq. (3.17). The results up to $N=5$ (filled circles) have been derived from the known values of the virial coefficients as given in Table I. while those for $N=6,7$, and 8 (open circles) have been obtained from the estimates of the higher virial coefficients given in Table [II] The open squares and diamonds represent the values of $\eta_{\infty}(N)$ when the estimates $b_{11}-b_{16}$ of Table III are assumed to have an increasing error between $\pm 10 \%$ and $\pm 30 \%(d=2)$ or between $\pm 20 \%$ and $\pm 60 \% \quad(d=3)$, as explained in the text. The horizontal lines correspond to $\eta_{\max }$. The curves correspond to the same fits as in Fig. 5.

feature that one can observe from Fig. 6 is that the new additions appear to continue the smooth trend obtained with the previous five points. In fact, they fall on top of the curve that served to fit these previous points in Fig. 5. Further, the last point of the curve corresponding to $d=3$ is above the random close-packing value $\eta_{\text {rcp }} \simeq 0.64$. Hence, all this evidence strongly supports the conjecture (1.6). However, a word of caution is called for since, irrespective of the tests that we have carried out on the sensitivity of $\eta_{\infty}(N)$ to errors in the values of the virial coefficients, the possibility that some of the estimates that we have used for the higher virial coefficients may be quite deficient does exist. One must recall that in higher dimensions there are negative virial coefficients 31 and, although it is unlikely that for hard spheres some of the coefficients $b_{11}$ to $b_{16}$ may be negative, one cannot rule it out with certainty.

Since the results of this section face the limitation of having been derived with the (relatively) small number of available virial coefficients, one may reasonably wonder whether our proposal would stand against a more stringent test. For instance, one could examine the model of hard hexagons on a lattice, for which an exact solution exists and where the first twenty five virial coefficients are given by Joyce $\stackrel{43}{=}$ or the model of hard squares on a lattice, where the first forty two virial coefficients can be obtained from the work by Baxter et al $\underline{\underline{44}}$ While the analysis (not shown but available upon request) carried out for these systems using the inverse Padé representation of the pressure similar to Eq. (3.17) is not incompatible with the validity of the conjecture (1.6), it presents large oscillations and is flawed by unphysical results arising from some defective Padé approximants. This may be due to different causes which we will discuss in connection with the hard hexagon model on a lattice. On the one hand, continuous and two-dimensional discrete models show important differences. For instance, in the hard hexagon model on a lattice there is no analytic continuation of the pressure from the low to the high density regime. In fact, the fluid branch and the solid branch both end and meet with a zero slope at the critical density in what appears to be a continuous phase transition from the disordered to the ordered state. In this case, no metastable states are possible. In contrast, computer simulations have revealed both for the hard-disk and the hard-sphere fluids that, in the thermodynamic limit, the low and high density branches of the equation of state do not smoothly join one another. In the case of hard disks, it is not clear yet whether the fluid-solid transition is first order or mediated by an intermediate hexatic phase. In the case of hard spheres, there exists compelling evidence that these systems present a first order phase transition where a two-phase region appears with a constant pressure as the density varies from the density of the pure fluid $\rho_{f}$ to the density of the pure solid $\rho_{s}$. The flat portion of the isotherm which connects $\rho_{f}$ and $\rho_{s}$ is the tie line. The fluid branch for $\rho>\rho_{f}$ is metastable but can be observed in computer simulations. Thus, while in the case of the hard hexagon model on a lattice one does know that the pressure diverges at the close-packing density in the solid branch, the question of where the analytic continuation of the fluid branch may diverge loses all meaning. On the other hand, as mentioned in Sec. I. for the hard-disk and hard-sphere fluids the existence of metastable states past the freezing point legitimately allows one to ask at which density this metastable fluid branch, when analytically continued, diverges. The present results suggest that knowledge of the virial coefficients may indeed indicate when this will occur for (continuous) hard-core fluids. 


\section{CONCLUDING REMARKS}

On the basis of our results, the following conclusions and remarks can be made. Two issues have been addressed in this paper. The first one concerns the question of whether the known virial coefficients (presently ten both for hard disks and hard spheres) are enough to tell us about the packing fraction $\eta_{\infty}$ at which the fluid equation of state, continued and extrapolated beyond the fluid-solid transition, has a divergence to infinity. In connection with this issue, the determination of $\eta_{\infty}$ from the direct Padé approximants [cf. Eq. (2.3)] seems to be not very reliable. Given the fact that as a thermodynamic variable the pressure has a clear physical meaning not shared by the compressibility factor, the inverse representation (3.17) appears then as the natural candidate for such a determination, at least when restricting to the presently available information, namely only the ten known virial coefficients.

The second issue is related to the validity of the conjecture (1.6). In the case of hard disks, all the results support it. On the other hand, strictly speaking, for the hard-sphere system our results allow us neither to validate nor to discard this conjecture. However, the robustness analysis carried out for hard spheres strongly suggests that the conjecture is true. Nevertheless, full confirmation must await the availability of higher virial coefficients. In any case, we are persuaded of the usefulness of the inverse representation (3.17) in order to finally clarify the issue.

\section{ACKNOWLEDGMENTS}

Two of us (M.A.G.M. and A.S.) acknowledge the financial support from the Ministerio de Ciencia e Innovación (Spain) through Grant No. FIS2010-16587 (partially financed by FEDER funds) and from the Junta de Extremadura (Spain) through Grant No. GR1015. The work of of M.R. and M.L.H. has been partially supported by DGAPA-UNAM under project IN-107010-2.

\footnotetext{
${ }^{1}$ M. Thiesen, Ann. Phys. 24, 467 (1885).

${ }^{2}$ H. Kamerlingh Onnes, Commun. Phys. Lab. Univ. Leiden 71 3 (1901); Proc. Koninkl. Acad. Wetensch., Amsterdam 4, 125 (1902).

${ }^{3}$ J. E. Mayer and M. G. Mayer, Statistical Mechanics (Wiley, NY, 1940), ch. 13.

${ }^{4}$ Theory and Simulation of Hard-Sphere Fluids and Related Systems, Lectures Notes in Physics, edited by A. Mulero (Springer, Berlin, 2008), Vol. 753.

${ }^{5}$ J. D. van der Waals, Proc. Koninkl. Acad. Wetensch., Amsterdam 1, 138 (1899).

${ }^{6}$ G. Jäger, Sitzber. Akad. Wiss. Wien Ber. Math. Natur-w. Kl. (Part 2a) 105, 15 (1896).

${ }^{7}$ L. Boltzmann, Sitzber. Akad. Wiss. Wien. Ber. Math. Natur-w. Kl. (Part 2a) 105, 695 (1896).

${ }^{8}$ J. J. van Laar, Proc. Koninkl. Acad. Wetensch., Amsterdam 1, 273 (1899).

${ }^{9}$ L. Boltzmann, Proc. Koninkl. Acad. Wetensch., Amsterdam 1, 398 (1899).

${ }^{10}$ L. Tonks, Phys. Rev. 50, 955 (1936).
}

${ }^{11}$ J. S. Rowlinson, Mol. Phys. 7, 593 (1964).

${ }^{12}$ P. C. Hemmer, J. Chem. Phys. 42, 1116 (1964).

${ }^{13}$ M. Luban and A. Baram, J. Chem. Phys. 76, 3233 (1982).

${ }^{14} \mathrm{M}$. Baus and J. L. Colot, Phys. Rev. A 36, 3912 (1987).

${ }^{15}$ N. Clisby and B. M. McCoy, J. Stat Phys. 114, 1343 (2004).

${ }^{16}$ I. Lyberg, J. Stat Phys. 119, 747 (2005).

${ }^{17}$ N. Metropolis, A. W. Rosenbluth, M. N. Rosenbluth, and A. H. Teller, J. Chem. Phys. 21, 1087 (1953).

${ }^{18}$ M. N. Rosenbluth and A. W. Rosenbluth, J. Chem. Phys. 22, 881 (1954).

${ }^{19}$ F. H. Ree and W. G. Hoover, J. Chem. Phys. 40, 939 (1964).

${ }^{20}$ F. H. Ree and W. G. Hoover, J. Chem. Phys. 41, 1635 (1964).

${ }^{21}$ J. S. Rowlinson, Rep. Progr. Phys. 28, 169 (1965).

${ }^{22}$ F. H. Ree and W. G. Hoover, J. Chem. Phys. 46, 4181 (1967).

${ }^{23}$ S. Kim and D. Henderson, Phys. Lett. A 27, 378 (1968).

${ }^{24}$ K. W. Kratky, Physica A 85, 607 (1976).

${ }^{25}$ K. W. Kratky, Physica A 87, 584 (1977).

${ }^{26}$ K. W. Kratky, J. Stat Phys. 27, 533 (1982).

${ }^{27}$ K. W. Kratky, J. Stat Phys. 29, 129 (1982).

${ }^{28}$ E. J. Janse van Rensburg and G. M. Torrie, J. Phys. A: Math. Gen. 26, 943 (1992).

${ }^{29}$ E. J. Janse van Rensburg, J. Phys. A: Math. Gen. 26, 4805 (1993).

${ }^{30}$ A. Y. Vlasov, X. M. You, and A. J. Masters, Mol. Phys. 100, 3313 (2002).

${ }^{31}$ N. Clisby and B. M. McCoy, J. Stat Phys. 114, 1361 (2004).

${ }^{32}$ S. Labík, J. Kolafa, and A. Malijevský, Phys. Rev. E 71, 021105 (2005).

${ }^{33}$ N. Clisby and B. M. McCoy, Pramana 64, 775 (2005).

${ }^{34}$ J. Kolafa and M. Rottner, Mol. Phys. 104, 3435 (2006).

${ }^{35}$ N. Clisby and B. M. McCoy, J. Stat Phys. 122, 15 (2006).

${ }^{36} \mathrm{M}$. Bishop, N. Clisby, and P. A. Whitlock, J. Chem. Phys. 128, 034506 (2008).

${ }^{37}$ B. M. McCoy, Advanced Statistical Mechanics (Oxford University Press, Oxford, 2010).

${ }^{38}$ J. L. Lebowitz and O. Penrose, J. Math. Phys. 5, 841 (1964).

${ }^{39}$ R. Fernández, A. Procacci and B. Scoppola, J. Stat Phys. 128, 1139 (2007)

${ }^{40}$ A. Santos and M. López de Haro, J. Chem. Phys. 130, 214104 (2009).

${ }^{41}$ V. C. Aguilera-Navarro, M. Fortes, M. de Llano, and O. Rojo, J. Chern. Phys. 81 1450, (1984).

${ }^{42}$ D. S. Gaunt and G. S. Joyce, J. Phys. A: Math. Gen. 13, L211 (1980).

${ }^{43}$ G. S. Joyce, Phil. Trans. R. Soc. London A 325, 643 (1988).

${ }^{44}$ R. J. Baxter, I. G. Enting, and S. K. Tsang, J. Stat. Phys. 22, 465 (1980).

${ }^{45}$ See http://www .math.rwth-aachen.de/ gabriele.nebe/lattices/ for a catalogue of lattices and a table with the densest packings presently known in dimensions up to 128 .

${ }^{46}$ I. C. Sanchez, J. Chem. Phys. 101, 7003 (1994).

${ }^{47}$ L. V. Woodcock, J. Chem. Soc. Faraday Trans. II 72, 731 (1976).

${ }^{48}$ F. C. Andrews, J. Chem. Phys. 62, 272 (1975); 64, 1941 (1976).

${ }^{49}$ A. Baram and M. Luban, J. Phys. C 12, L659 (1979).

${ }^{50}$ J. A. Devore and E. Schneider, J. Chem. Phys. 77, 1067 (1982).

${ }^{51}$ V. C. Aguilera-Navarro, M. Fortes, M. de Llano, A. Plastino, and O. Rojo, J. Stat Phys. 32, 95 (1983).

${ }^{52}$ B. R. Hoste and W. van Dael, J. Chem. Soc. Faraday Trans. II 80, 477 (1984).

${ }^{53}$ J. I. Goldman and J. A. White, J. Chem. Phys. 89, 6403 (1988).

${ }^{54}$ A. Santos, M. López de Haro, and S. B. Yuste, J. Chem. Phys. 103, 4622 (1995).

${ }^{55}$ W. Wang, M. K. Khoshkbarchi, and J. H. Vera, Fluid Phase Equil. 115, 25 (1996).

${ }^{56}$ M. K. Khoshkbarchi and J. H. Vera, Fluid Phase Equil. 130, 189 (1997).

${ }^{57}$ Kh. Nasrifar, Sh. Ayatollahi, and M. Moshfeghian, Can. J. Chem. Eng. 78, 1111 (2000).

${ }^{58}$ C. Ghotbi and J. H. Vera, Can. J. Chem. Eng. 79, 678 (2001).

${ }^{59}$ X. Z. Wang, Phys. Rev. E 66, 31203 (2002). 
${ }^{60}$ I. Polishuk and J. H. Vera, J. Phys. Chem. B 109, 5977 (2005).

${ }^{61}$ M. Miandehy, H. Modarress, and M. R. Dehghani, Fluid Phase Equil. 239, 91 (2006).

${ }^{62}$ D. I. Korteweg, Nature 45, 277 (1892).

${ }^{63}$ L. Boltzmann, Vorlesungen über Gastheorie (Barth, Leipzig, 1898), Vol. 2, Chap. 5 [Lectures on Gas Theory, Part 2, translated by S. G. Brush (University of California Press, Berkeley, CA, 1964, 2006), Chap. 5 (in English)].

${ }^{64}$ E. J. Le Fevre, Nature (London) Phys. Sci. 235, 20 (1972).

${ }^{65}$ V. C. Aguilera-Navarro, M. Fortes, M. de Llano, and A. Plastino, J. Chem. Phys. 76, 749 (1982).

${ }^{66}$ D. Ma and G. Ahmadi, J. Chem. Phys. 84, 3449 (1986).

${ }^{67}$ Y. Song, R. M. Stratt, and E. A. Mason, J. Chem. Phys. 88, 1126 (1988).

${ }^{68}$ E. Z. Hamad, Ind. Eng. Chem. Res. 36, 4385 (1997).

${ }^{69}$ J. G. Berryman, Phys. Rev. A 27, 1053 (1983).

${ }^{70}$ A. Mulero, C. A. Galán, M. I. Parra, and F. Cuadros, in The- ory and Simulation of Hard-Sphere Fluids and Related Systems, Lectures Notes in Physics, edited by A. Mulero (Springer, Berlin, 2008), Vol. 753, pp. 37-109.

${ }^{71}$ I. C. Sanchez and J. S. Lee, J. Phys. Chem. B. 113, 15572 (2009).

${ }^{72}$ C. Bender and S. Orszag, Advanced Mathematical Methods for Scientists and Engineers (McGraw-Hill, NY, 1978), ch. 8.

${ }^{73}$ A. O. Guerrero and A. Bassi, J. Chem. Phys. 129, 044509 (2008).

${ }^{74}$ J. Hu and Y. Yu, Phys. Chem. Chem. Phys. 11, 9382 (2009).

${ }^{75}$ E. Z. Hamad and G. O. Yahaya, Fluid Phase Equilib. 168 59, (2000).

${ }^{76}$ Z. W. Salsburg, R. W. Zwanzig, and J. G. Kirkwood, J. Chem. Phys. 21, 1098 (1953).

${ }_{77}$ D. S. Corti and P. G. Debenedetti, Phys. Rev. E 57, 4211 (1998). ${ }^{78}$ M. Heying and D. S. Corti, Fluid Phase Equilib. 220, 85 (2004).

${ }^{79}$ R. Fantoni, A. Giacometti, Al. Malijevský, and A. Santos, J. Chem. Phys. 131, 124106 (2009).

${ }^{80}$ A. Ben-Naim and A. Santos, J. Chem. Phys. 131, 164512 (2009). 